\title{
AN EXTENSION OF THE KAHANE-KHINCHINE INEQUALITY
}

\author{
DAVID C. ULLRICH
}

Let $\omega_{1}, \ldots, \omega_{2}, \ldots$, denote the Steinhaus variables: independent identically distributed random variables, uniformly distributed on $[0,1]$.

THEOREM. There exists $c>0$ such that if $x_{1}, \ldots, x_{N}$ are elements of any (complex) Banach space $B$ then

$$
\exp \mathrm{E} \log \left\|\sum_{j=1}^{N} e^{2 \pi i \omega_{j}} x_{j}\right\| \geq c\left\{\mathrm{E}\left\|\sum_{j=1}^{N} e^{2 \pi i \omega_{j}} x_{j}\right\|^{2}\right\}^{1 / 2} .
$$

(Here "exp" is the exponential, and "E" denotes "expected value".)

The Kahane-Khinchine inequality (see [KH, Chapter 2, Theorem 4, or AG, p. 176] for the original proof; another argument due to C. Borell may be found in [BK or LT, Theorem 1.e.13]) states that

$$
\left\{\mathrm{E}\left\|\sum_{j=1}^{N} e^{2 \pi i \omega_{j}} x_{j}\right\|^{p}\right\}^{1 / p} \geq c_{p}\left\{\mathrm{E}\left\|\sum_{j=1}^{N} e^{2 \pi i \omega_{j}} x_{j}\right\|^{2}\right\}^{1 / 2} \quad(p>0) .
$$

Recalling that in general $\left\{E|f|^{p}\right\}^{1 / p}$ decreases to $\exp E \log |f|$ as $p$ decreases to zero, one sees that (1) is a strictly stronger statement than (2); in fact (1) says simply that $c_{p}$ may be taken bounded away from zero in (2). Note that the inequality obtained from (1) by replacing $e^{2 \pi i \omega_{j}}$ with the $j$ th Rademacher function $r_{j}$ is false, even in the case $B=\mathrm{C}$ : If $s_{n}=n^{-1 / 2}\left(r_{1}+\cdots+r_{n}\right)$ then $\exp E \log \left|s_{n}\right|=0$ for even values of $n$, although $s_{n}$ is asymptotically normal. In other words: Suppose that $X$ is a random variable; suppose even $|X| \leq 1$ a.s. Then to say $\exp E \log |X| \geq c$ implies that the set where $X$ is small must be small, while to say $\left\{\mathrm{E}|X|^{p}\right\}^{1 / p} \geq c$ does not even preclude the possibility that $X$ vanish on a set of positive measure!

In the case $B=\mathbf{C}$ inequality ( 1 ) is proved in [UK], and various applications are given. In particular one may use (1) to show that the zero set of a Bloch function may be strictly larger than is possible for a function in the "little-oh" Bloch space, answering a question of Ahern and Rudin [AR]; this fact then gives a result analogous to Theorem 6.1 of $[\mathbf{A R}]$, with VMOA and $H^{\infty}$ replaced by BMOA and VMOA, respectively. Inequality (1) also allows one to construct new and improved Ryll-Wojtaszczyk polynomials [RW]: There exists a sequence $P_{1}, P_{2}, \ldots$, of polynomials in $\mathbf{C}^{n}$ such that $P_{j}$ is homogeneous of degree $j$ and satisfies $\left|P_{j}(z)\right| \leq 1\left(z \in \mathbf{C}^{n},|z| \leq 1\right)$ while

$$
\exp \int_{S} \log \left|P_{j}\right| d \sigma \geq c>0 \text {. }
$$

Received by the editors June 24, 1987.

1980 Mathematics Subject Classification (1985 Revision). Primary 60G50, 42C10. 
Here $\sigma$ is normalized Lebesgue measure on $S$, the unit sphere in $\mathbf{C}^{n}$. (Ryll and Wojtaszczyk give

$$
\int_{S}\left|P_{j}\right|^{2} d \sigma \geq c
$$

in place of (3).)

Now let $\tilde{\omega}_{1}, \tilde{\omega}_{2}, \ldots$, be a second sequence of Steinhaus variables, independent of the $\omega_{1}, \omega_{2}, \ldots$. It is not too difficult to see that our theorem implies

(5) $\exp \mathrm{E} \log \left\|\sum_{j, k=1}^{N} e^{2 \pi i \omega_{j}} e^{2 \pi i \tilde{\omega}_{k}} x_{j, k}\right\| \geq c\left\{\mathrm{E}\left\|\sum_{j, k=1}^{N} e^{2 \pi i \omega_{j}} e^{2 \pi i \tilde{\omega}_{k}} x_{j, k}\right\|^{2}\right\}^{1 / 2}$

for $x_{j, k} \in B(1 \leq j, k \leq N)$. (And similarly for $n$ mutually independent sequences of Steinhaus variables, by induction.) The proof involves applying (1) in a certain space of square-integrable $B$-valued random variables; thus it would appear that even the special case of (5) corresponding to $B=\mathbf{C}$ does not follow directly from results in [UK], but rather constitutes an application of the present "vector-valued" inequality to the scalar-valued case.

We would like to give an idea of the proof of (1):

Suppose that $\mathrm{E}\left\|\sum_{j=1}^{N} e^{2 \pi i \omega_{j}} x_{j}\right\|^{2}=1$, and define

$$
\Psi(\lambda)=P\left(\left\|\sum_{j=1}^{N} e^{2 \pi i \omega_{j}} x_{j}\right\|<\lambda\right)
$$

for $\lambda>0$. We need only show that

$$
\int_{0}^{1} \Psi(\lambda) \frac{d \lambda}{\lambda} \leq c .
$$

Take $\left\|x_{1}\right\| \geq\left\|x_{j}\right\|$ for all $j$. For $0<\lambda<\left\|x_{1}\right\| / 2$ the triangle inequality shows that

$$
P\left(\left\|e^{2 \pi i \omega_{1}} x_{1}+y\right\|<\lambda\right) \leq c \lambda /\left\|x_{1}\right\|
$$

for any $y \in B$; this gives

$$
\int_{0}^{\left\|x_{1}\right\| / 2} \Psi(\lambda) \frac{d \lambda}{\lambda} \leq c
$$

by independence. Since $\Psi(\lambda) \leq 1$, (8) leads to

$$
\int_{0}^{K\left\|x_{1}\right\|} \Psi(\lambda) \frac{d \lambda}{\lambda} \leq c
$$

for any fixed $K$. Inspired by Theorem 3 in Chapter 2 of $[\mathbf{K H}]$ (or see inequality 2.5 on p. 106 of [AG]) we were able to prove a sort of "concentration inequality":

LEMMA. If $K$ is large enough then there exists $\gamma \in(0,1)$ such that if $K\left\|x_{1}\right\| \leq \lambda \leq 1$ then $\Psi(\gamma \lambda) \leq \frac{1}{2} \Psi(\lambda)$.

It is easy to see that the lemma implies

$$
\int_{K\left\|x_{1}\right\|}^{1} \Psi(\lambda) \frac{d \lambda}{\lambda} \leq c
$$


certainly (9) and (10) give (7).

Note that in the case $B=C$ one may use the Fourier transform (the "method of characteristic functions") to establish (10) more simply; see [UK].

\section{REFERENCES}

[AG] A. Araujo and E. Gine, The central limit theorem for real and Banach valued random variables, Wiley \& Sons, New York, 1980.

[AR] P. Ahern and W. Rudin, Bloch functions, BMO, and boundary zeroes, Indiana Math. J. 36 (1987), 131-148.

[BK] C. Borell, $A$ note on the integrability of Walsh polynomials (unpublished).

[KH] J. P. Kahane, Some random series of functions, D. C. Heath \& Co., Lexington, Mass., 1968.

[LT] J. Lindenstrauss and L. Tzafriri, Classical Banach spaces, vol. II, Springer-Verlag, New York, 1979.

[RW] J. Ryll and P. Wojtaszczyk, On homogeneous polynomials on a complex ball, Trans. Amer. Math. Soc. 276 (1983), 107-116.

[UB] D. Ullrich, An extension of the Kahane-Khinchine inequality in a Banach space (to appear).

[UK] _ Khinchine's inequality and the zeroes of Bloch functions (to appear).

Department of Mathematics, OKLAhoma State University, Stillwater, OKLAHOMA 74078 